\title{
Challenges and Benefits of Utilizing Carbon Nanofilaments in Cementitious Materials
}

\author{
Ardavan Yazdanbakhsh, Zachary Grasley, Bryan Tyson, and Rashid Abu Al-Rub \\ Zachry Department of Civil Engineering, Texas A\&M University, CE/TTI 503-C, 3136 TAMU, College Station, \\ TX 77843-3136, USA \\ Correspondence should be addressed to Zachary Grasley, zgrasley@civil.tamu.edu
}

Received 14 January 2012; Accepted 18 April 2012

Academic Editor: Tong Lin

Copyright ( 92012 Ardavan Yazdanbakhsh et al. This is an open access article distributed under the Creative Commons Attribution License, which permits unrestricted use, distribution, and reproduction in any medium, provided the original work is properly cited.

\begin{abstract}
Carbon nanofibers/tubes (CNF/Ts) are very strong and stiff and as a result, are expected to be capable of enhancing the mechanical properties of cementitious materials significantly. Yet there are practical issues concerning the utilization of CNF/Ts in cementitious materials. This study summarizes some of the past efforts made by different investigators for utilizing carbon nanofilaments in cementitious materials and also reports recent experimental research performed by the authors on the mechanical properties of CNF-reinforced hardened cement paste. The major difficulties concerning the utilization of CNF/Ts in cementitious materials are introduced and discussed. Most of these difficulties are related to the poor dispersibility of CNF/Ts. However, the findings from the research presented in this work indicate that, despite these difficulties, carbon nanofilaments can significantly improve the mechanical properties of cementitious materials. The results show that CNFs, even when poorly dispersed within the cementitious matrix, can remarkably increase the flexural strength and cracking resistance of concrete subjected to drying conditions.
\end{abstract}

\section{Introduction}

Due to their excellent mechanical properties, carbon nanofibers/tubes $(\mathrm{CNF} / \mathrm{Ts})$ have been the subject of many investigations in the past decade where they have been used as inclusions in composite materials. Mechanically, CNTs exhibit elastic Young's moduli of more than $1 \mathrm{TPa}(1.5 \times$ $10^{8} \mathrm{psi}$ ) [1]. Their theoretical strength is 100 times that of steel, at only $1 / 6$ th the specific gravity [2]. Values as high as $60 \mathrm{GPa}\left(8.7 \times 10^{6} \mathrm{psi}\right)$ for ultimate strength and $6 \%$ for ultimate strain have been reported $[3,4]$. Salvetat et al. reported an elastic strain capacity of $12 \%$, which is 60 times higher than that of steel [1]. CNTs are also highly flexible, being capable of bending in circles or forming knots. Like macroscopic tubes, they can buckle or flatten under appropriate loadings [5]. Yakobson and Avouris further summarize the mechanical behavior of CNTs [6]. CNTs are extremely small and their diameter is usually less than $20 \mathrm{~nm}$. CNFs, on the other hand, are relatively large; their diameter can be as large as $200 \mathrm{~nm}$. Recently, Ozkan et al. performed direct mechanical measurements on CNFs [7]. The CNFs that they investigated had a tensile strength between 2 and $5 \mathrm{GPa}\left(2.9 \times 10^{5}-7.3 \times 10^{5} \mathrm{psi}\right)$ with an average Young's modulus of elasticity of $300 \mathrm{GPa}\left(4.4 \times 10^{7} \mathrm{psi}\right)$.

Many studies have been carried out in the past decade regarding the incorporation of carbon nanofilaments in cementitious materials. Comparing the experimental results from those studies shows that the findings regarding the effect of CNF/Ts on the mechanical properties of cementitious materials are hardly conclusive $[8,9]$. There are two main reasons for the existing inconsistencies: first, the utilization of different types of $\mathrm{CNF} / \mathrm{Ts}$ and, second, the adoption of different mixing and dispersion methods. Carbon nanofilaments are available in a wide range of sizes; their diameter can be between $1 \mathrm{~nm}$ and $200 \mathrm{~nm}$ and their length ranges from less than a micrometer to over a millimeter. Van der Waal's forces are more effective in causing the agglomeration of smaller particles. Moreover, filaments with higher aspect ratios tend to tangle easier and form clumps. In addition, different $\mathrm{CNF} / \mathrm{Ts}$ have different surface properties (including surface free energies) that affect their dispersibility. Investigators have used several 
different approaches to incorporate CNF/Ts in cementitious materials. Most of them are shown to be insufficiently effective in dispersing the nanofilaments uniformly within cementitious matrices. Poor dispersion of the nanofilaments in cementitious matrices is a potential reason for the usually modest effect of CNF/Ts on the strength of the cementitious nanocomposites.

Almost any fiber that is utilized for reinforcing and enhancing concrete mechanical response is designed for this purpose; its material, shape, and size are designed and optimized so that by incorporating them in cementitious matrices the desired properties are achieved. As opposed to such custom-made fibers, CNF/Ts are not specifically made to be used in cementitious materials such as concrete. The primary reason that has motivated the scientists to research the potentials of $\mathrm{CNF} / \mathrm{Ts}$ for enhancing concrete is the unique properties of these nanofilaments.

Past research efforts on CNF/T-incorporated cementitious materials are mostly experimental in nature with little or no analytical and theoretical basis towards engineering $\mathrm{CNF}$ /T-incorporated cementitious materials; the investigators procure some carbon nanofilaments, incorporate them in a fresh cementitious material, cast some specimens, and test them to observe the effect of carbon nanofilaments on different properties. This type of research, despite not being fundamental, has led to important findings regarding the existing challenges that need to be considered when analytically predicting the behavior of CNF/T-incorporated cementitious materials. For example, in simulations and theoretical analyses for predicting the effect of $\mathrm{CNF} / \mathrm{Ts}$ on strength of materials in which they are incorporated, one cannot always assume that the nanofilaments are randomly dispersed. In other words, the analytical researcher should be cautioned that in practice it is extremely difficult to achieve a random dispersion of carbon nanofilaments in a cementitious matrix.

The objective of the present paper is to highlight the issue of dispersion of carbon nanofilaments in cementitious materials and to demonstrate the effect of well-and poorly dispersed CNFs on the strength and crack resistance of hardened cement paste under different curing conditions. Some of the existing methods for mitigating the poor dispersion of CNF/Ts and the possible shortcomings of those methods will be discussed. Past efforts on the incorporation of $\mathrm{CNF} / \mathrm{Ts}$ in cementitious materials will be reviewed and new results presented.

\subsection{Dispersion of CNF/Ts in Cementitious Matrices. CNT/Fs} strongly attract each other due to van der Waal's forces. This attraction results in the formation of agglomerations that are very difficult to disentangle. The dispersion problem has been combated by methods like surface modification of the fibers and by using surfactants, usually in combination with ultrasonic processing of the nanofilaments in liquid solutions [10-16]. Although different methods such as implanting or growing the fibers directly on nonhydrated cement grains are being studied by some investigators [17-19], the most common method of producing CNT/F-incorporated cement paste is to first disperse the nanofilaments in water, typically by using surfactants and ultrasonic processing, and then mix the aqueous dispersion with cement. This method will be referred to as the "ultrasonication method" in the current paper. Unfortunately, most of the effective surfactants are not compatible with cement hydration and their presence in cement paste results in a weak material, usually entrapping a notable amount of air [9]. Therefore, to avoid negative hydration and air entrapment issues, weaker surfactants known as water reducing admixtures or superplasticizers are used. These surfactants are typically polycarboxylate based and are specifically made for cementitious materials. They are typically added to a fresh mix of cementitious material to disagglomerate the cement grains and disperse them, thereby reducing the amount of water required to produce a paste with a certain rheological property.

It has been shown that the ultrasonication method does not maintain a stable dispersion of nanofilaments in cementitious materials and large volumes of the hardened paste can remain absent of nanofilaments [9]. The reason, as shown in a study by and Grasley and Yazdanbakhsh [20], is that nanofilaments can move freely in fresh cement paste and van der Waals attracting forces, although partially counterbalanced with the surfactant effect, will eventually cause the initially dispersed nanofilaments to migrate and reagglomerate. It is not yet clearly understood why the carbon nanofilaments that can remain well dispersed for days or even months in a water-superplasticizer solution reagglomerate relatively rapidly when the solution is added to cement. However, it is definitely known that the reagglomeration issue of carbon nanofilaments in the matrix of host material (sometimes even in polymeric nanocomposites [21]) does exist.

One reason the free movement and therefore the reagglomeration of CNFs in fresh cement paste are possible is that most cement grains and the spacing between them are much larger than CNFs. As a result, there are large water-filled volumes between cement grains that impose little resistance on the movement of nanofilaments. If the space between the cement grains can be partially filled by welldispersed and stable secondary nanoparticles, the movement of carbon nanofilaments will be confined. This concept has been implemented in the past to produce a stable dispersion of CNTs in polymeric materials. For example, secondary particles, such as clay, that have been used to improve electrical conductivity in polymer composites containing vapor grown carbon fibers [22] or carbon black [23] as the conductive filler, were also found to improve the dispersion of filaments. Liu and Grunlan used nanoparticles of clay to improve the dispersion of carbon nanotubes in epoxy composites [24]. Sanchez and Ince [25] were amongst the first to utilize silica fume as a means to improve the dispersion of carbon nanofilaments in cementitious materials. Recently, Yazdanbakhsh and Grasley showed that the combined utilization of silica fume and superplasticizer can significantly enhance and stabilize the dispersion of CNFs in cement paste [20].

Silica fume is an amorphous submicron powder (with particles 100 to 150 times smaller than a grain of cement) 
used to enhance several properties of concrete such as compressive strength, bond strength, and abrasion resistance $[26,27]$ and also to reduce permeability [28]. Mixture proportions for high-strength concrete typically contain 5 to 15 percent silica fume by mass of cement [29]. The method introduced by Yazdanbakhsh and Grasley for producing CNF-incorporated cement paste using silica fume as a stabilizer is a simple extension to the ultrasonication method. First, a water-superplasticizer solution is made. Then, CNFs are added to the solution and mixed manually. The solution is ultrasonicated and then added to the mixer that contains dry cement. Finally, silica fume is added during the mixing process.

It should be mentioned that the procedure involving utilizing silica fume could effectively disperse and stabilize the dispersion of the type of carbon nanofilaments used by Yazdanbakhsh and Grasley, who have used ball-milled CNFs. Ball milling breaks the CNF clumps and as a result makes the fibers more dispersible. Furthermore, since CNFs are larger than CNTs, they are also more dispersible. In general, the effectiveness of any dispersion and mixing method is highly related to the type of nanofilaments utilized. As will be seen in Section 2.2, there is an issue inherent in utilizing silica fume as a dispersion stabilizer. As received silica fume is typically clumped. Moreover, a high concentration of silica fume (over 15\% of the cement weight) is required to maintain a relatively uniform dispersion in a cementitious matrix. Therefore, the cementitious material will contain many nonreacted silica fume clumps that may act as crack initiation zones.

1.2. Mechanical Properties of CNF/T-Reinforced Cementitious Materials. Kowald used CNTs in cement paste with $\mathrm{CNT} /$ cement weight ratios in the range of 0.5 to $5.0 \%$ [30]. He tested the hardened specimens for compressive strength after 7, 14, and 28 days. Marginal improvements were observed in compressive strength and even a decrease in strength when the fiber dose was as high as $2.5 \%$ or more. $\mathrm{Li}$ et al. performed a set of experiments with CNTs in mortars with $\mathrm{CNT} /$ cement weight ratio of $0.5 \%$ [31]. The bending and compression tests showed that the addition of CNTs increased the compressive and flexural strength by $19 \%$ and $25 \%$, respectively. More references about the investigations performed on CNF/T-incorporated cementitious materials can be found in $[8,9]$. Gay and Sanchez tested hardened cement paste specimens with different compositions and found that the addition of $0.2 \%$ CNFs per weight of cement resulted in increased splitting tensile strength of $22 \%$ in portland cement composites and $26 \%$ in cementitious composites that also contained silica fume [32]. Metaxa et al. [33], Shah [34], and Konsta-Gdoutos et al. [35] showed that CNTs in cement matrix $(\mathrm{w} / \mathrm{c}=0.5)$ increased the flexural strength and the Young's modulus of plain cement paste by $25 \%$ and $50 \%$, respectively. (In the notation of concrete and cementitious materials research and industry, w/c is the ratio of the weight of water to the weight of cement used to produce the cementitious material. When pozzolanic materials such as fly ash or silica fume are utilized in addition to cement, the notation $\mathrm{w} / \mathrm{cm}$ is used, where $\mathrm{cm}$ represents the sum of the weights of cement and pozzolanic materials.) In another investigation, Konsta-Gdoutos et al. reported that the flexural strength of the specimens reinforced with CNTs shows an increase of $30-40 \%$ over plain cement specimens [36]. Cwirzen et al. reported an increase of $50 \%$ in the compressive strength of hardened cement paste due to the use of CNTs with a concentration of $0.045 \%$ to the weight of cement [37].

Recently, an investigation was performed by Tyson et al. regarding the effect of $\mathrm{CNF} / \mathrm{Ts}$ on the mechanical properties of hardened cement paste [38]. The results showed that utilizing CNFs with only a $\mathrm{CNF} / \mathrm{c}$ weight ratio of $0.1 \%$ increased the flexural strength of hardened cement paste by over $80 \%$, although the dispersion of CNFs in cement paste was not uniform. In light of the results from other investigations, this finding was remarkable and motivated the experiments reported in this section.

\section{The Effect of Carbon Nanofilaments on the Strength and Crack Resistance of Cementitious Materials}

This section reports on an experimental investigation of the effect of CNF reinforcement on flexural strength and crack resistance of hardened cement paste, with varying mix proportions and curing conditions. Normal strength and high-strength cement pastes in both plain and CNFreinforced forms were produced and exposed to two different curing conditions. In addition, in some of the batches silica fume was used as a dispersion stabilizer to observe whether the resulting enhanced dispersion can render CNFs more effective in improving the mechanical properties of hardened cement paste.

2.1. Experimental Program. Tyson et al. [38], Kowald [30], and Konsta-Gdoutos et al. [36] each found that after the CNF concentration exceeded a certain limit, CNFs became less effective in improving mechanical properties such as flexural strength. In fact, they observed a significant decline in flexural strength when the CNF to cement weight ratio $(\mathrm{CNF} / \mathrm{c})$ was increased from $0.1 \%$ to $0.2 \%$, most likely due to the poor dispersion of CNFs. In this study, in order to enhance dispersion in comparison to that achieved in past studies, a larger dosage of superplasticizer and more prolonged ultrasonic processing and paste mixing were utilized. To reduce variability, beams with cross-sectional areas larger than those made by Tyson et al. [38] were tested. Bending tests were performed on the beams to measure flexural strength, Young's modulus, and resilience. In addition, the effect of CNFs on the shrinkage cracking of beams was observed.

2.1.1. Materials and Instruments. The CNFs used in this experiment have a diameter between 60 and $150 \mathrm{~nm}$, a length between 30 and $100 \mu \mathrm{m}$, and specific surface area of $50-60 \mathrm{~m}^{2} / \mathrm{g}$. The CNFs were provided by Applied Sciences 
Incorporated under the commercial name Pyrograph PR-24XT-PS. The surfactant used for dispersing CNFs in water was a high-range polycarboxylate-based water reducing admixture (superplasticizer) provided by W. R. Grace with the commercial name ADVA Cast 575. Type I portland cement was used for producing cement paste.

Aqueous dispersions were generated by ultrasonically processing the CNFs in a water-superplasticizer solution. A $20 \mathrm{kHz}$ sonicator with a $12.5 \mathrm{~mm}$ (1/2 in.) diameter titanium alloy probe was used at an amplitude setting of $50 \%$. To produce high speed and high shear mixing, a $600 \mathrm{~W}$ Oster BVCB07-Z blender was used for mixing cement paste at approximately 7500 RPM. An optical microscope was used to image CNFs in aqueous dispersions and fresh cement paste using transmission mode. For this purpose, a Zeiss Axiophot microscope was used with 40x and 100x objectives lenses. A Bose ElectroForce 3230-AT System was used for four-point bending tests. The tests were performed in the load-controlled mode and the load-deflection values were registered until fracture occurred. A loading rate of $5 \mathrm{~N} / \mathrm{s}$ was applied, which was sufficiently slow so that it took at least few minutes for each beam to reach its ultimate strength, and the loading could be regarded as essentially static.

\subsubsection{Mix Proportions, Preparation of Specimens, and Testing.} The mix proportions tested in this study are presented in Table 1. To observe the possible impact of w/c on the effectiveness of CNFs in changing the mechanical properties, for the batches without silica fume, w/c of 0.25 and 0.40 were investigated (batches 1, 2, 6, and 7). Silica fume to cement weight ratio of 0.20 was used in batches 8 and 9. In those batches the $\mathrm{w} / \mathrm{cm}$ was 0.40 . To maintain a consistent viscosity, in the batches containing CNF or silica fume, the superplastilizer was used with the concentrations ranging from $0.66 \%$ to $1.42 \%$ to the weight of cement. The workability of the pastes was quantified based on the amount of the power consumed by the blender during mixing, which was monitored using an electricity power (i.e., wattage) monitor. This method was implemented by first recording the power consumed for plain cement paste with w/c of 0.40 , which had a desirable viscosity, and then using an amount of superplasticizer in other batches that resulted in the same or similar value of power consumption. Since superplasticizer should not be added to the paste during paste mixing and is required to be added to the aqueous solution to yield the best possible dispersion of CNFs in water during ultrasonic processing, a pilot experimental study was performed in which several batches of cement paste were made with the compositions presented in Table 1 to determine the required amount of superplasticizer for each mix composition. It should be noted that all the batches had the same volume.

As mentioned previously, in past studies found in the literature, at some high level of CNF concentration the measured strength generally began to decline due to poor dispersion. In order to test the ability of the modified manufacturing process utilized in this project, CNF concentrations that would be expected to cause strength reductions utilizing past preparation techniques were considered. For
TABle 1: Mix proportion of the cement paste batches. ${ }^{*}$ In batch 5 CNFs were directly added, in form of dry powder, to cement paste in the mixer, while in the other batches CNFs were first dispersed in a water-superplasticizer solution using ultrasonic processing.

\begin{tabular}{lccccc}
\hline $\begin{array}{l}\text { Batch } \\
\text { no. }\end{array}$ & $\begin{array}{c}\text { CNF } \\
(\mathrm{cm})\end{array}$ & $\begin{array}{c}\text { W } \\
(\mathrm{cm})\end{array}$ & $\begin{array}{c}\text { Silica fume } \\
(\mathrm{c})\end{array}$ & $\begin{array}{c}\text { Superplasticizer } \\
(\mathrm{cm})\end{array}$ & $\begin{array}{c}\text { Cast in } \\
100 \% \\
\mathrm{RH}\end{array}$ \\
\hline 1 & 0.00 & 0.40 & 0.00 & 0.00 & No \\
2 & 1.00 & 0.40 & 0.00 & 0.74 & No \\
3 & 0.00 & 0.40 & 0.00 & 0.00 & Yes \\
4 & 1.00 & 0.40 & 0.00 & 0.74 & Yes \\
$5^{*}$ & 1.00 & 0.40 & 0.00 & 0.74 & Yes \\
6 & 0.00 & 0.25 & 0.00 & 0.66 & No \\
7 & 1.00 & 0.25 & 0.00 & 0.85 & No \\
8 & 0.00 & 0.40 & 0.20 & 0.67 & Yes \\
9 & 1.00 & 0.40 & 0.20 & 1.42 & Yes \\
\hline
\end{tabular}

this purpose, the $\mathrm{CNF} / \mathrm{cm}$ weight ratio ( $\mathrm{cm}$ indicates the sum of the weights of cement and silica fume, if any) of 1.0\% was selected to be used in all the CNF-incorporated cement paste batches. Preliminary investigations showed that, if higher concentrations were selected, CNFs were very difficult to disperse in the aqueous solution even by using large amounts of superplasticizer which intrinsically causes issues such as entrapping excessive air in the paste and decelerating cement hydration.

To produce each cement paste, first superplasticizer was mixed with water. Then CNFs were added to the solution and mixed with a manual stirrer for one minute. The mixture was then sonicated for 10 minutes. The resulting aqueous dispersion was then added to the blender that contained cement and mixed for 10 minutes. For the batches with silica fume, the aqueous dispersion was added gradually to the paste in the blender during mixing; this made the mixing process easier and prevented the formation of dry clumps of cement/silica fume in the paste at the beginning of mixing. A similar procedure was used for making the batches with $\mathrm{w} / \mathrm{c}$ of 0.25 ; half of the cement was placed in the mixer before starting mixing, and the other half was added to the paste during mixing.

After mixing was completed, the fresh paste was cast in PVC molds. Each mold had a square cross-section with the side length of $15.9 \mathrm{~mm}$ ( $0.625 \mathrm{inch})$ and length of $240 \mathrm{~mm}$ (9.5 in). To study the effect of CNFs on the early age shrinkage cracking, as indicated in Table 1, for some batches $(1,2,6$, and 7$)$, after casting the beams the mold was kept in the lab at room temperature $\left(25^{\circ} \mathrm{C}\right)$ and relative humidity (RH) (approximately 65\%) for $24 \mathrm{hrs}$. After this $24 \mathrm{hr}$ period, the beams were demolded and transferred to a humidity chamber with an RH of $100 \%$ and kept there until testing. The rest of the beams were cast in the humidity chamber and were maintained at $100 \% \mathrm{RH}$ until testing.

For each batch, 8 beams were cast and 5 of them were tested so that the results could be averaged. More beams were tested when there were outliers (typically, beams with flexural strength of less than half of the average flexural 


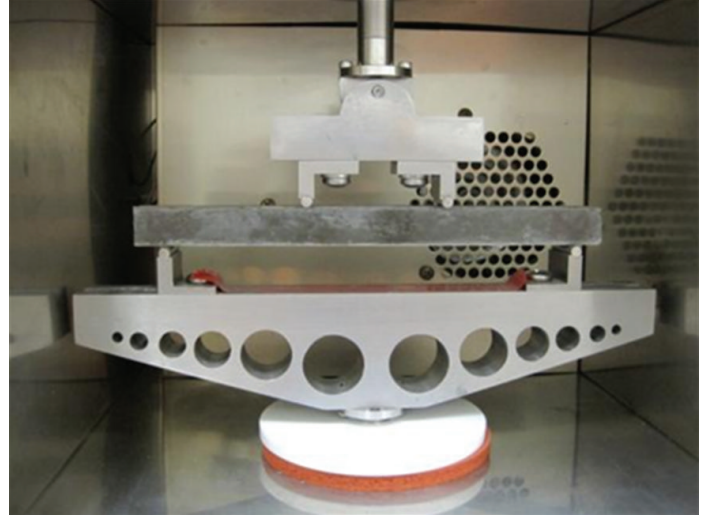

FIGURE 1: Four-point bending setup for testing hardened cement paste beam specimens. The beam has a cross-section with the side length of $15.9 \mathrm{~mm}$ (0.625 inch) and length of $240 \mathrm{~mm}$ (9.5 in).

strength value). The outliers were disregarded. The beams were tested at an age of 45 days. This period was chosen for curing instead of 28 days to offset the retarding effect of superplasticizers on hydration since different concentrations of superplasticizer were utilized for producing different batches. The beams were removed from the humidity chamber, towel-dried, and tested after 30 minutes in fourpoint bending setup. The test setup is shown in Figure 1 . The span between supports was $180 \mathrm{~mm}$ (7 in). The location of the supports and load points divided the beam span into three equal segments. The beams were tested in loadcontrolled mode and the values of applied load and beam midspan deflection were recorded until the beam fractured. As mentioned earlier, load was applied with the rate of $5 \mathrm{~N} / \mathrm{s}$.

2.1.3. Calculations. Three properties of each beam were determined from the bending tests: flexural strength, Young's modulus, and resilience. Simple beam theory was utilized to calculate these properties. Flexural strength (or maximum tensile stress in the lower fiber of the beam under loading) was calculated by

$$
\sigma_{\max }=\frac{F \cdot L}{b \cdot h^{2}},
$$

where $F$ is the applied force, $(F / 2$ is applied by each of the two load points), $L$ is the beam span, and $b$ and $h$ are beam's width and height, respectively. Young's modulus was calculated by

$$
E=k \frac{F}{\delta}
$$

where $\delta$ is the displacement of the beam midspan due to the application of $F \cdot(F / \delta)$ is the slope of the elastic portion of load versus deflection curve, and $k$ is a constant value for beams of the same dimension and span. $k$ was calculated by

$$
k=\frac{23}{1296} \frac{L^{3}}{I}
$$

TABLE 2: Mechanical properties of the tested beams.

\begin{tabular}{lccc}
\hline $\begin{array}{l}\text { Batch } \\
\text { no. }\end{array}$ & $\begin{array}{c}\text { Flexural strength, } \\
(\mathrm{MPa})\end{array}$ & $\begin{array}{c}\text { Young's modulus, } \\
(\mathrm{GPa})\end{array}$ & $\begin{array}{c}\text { Resilience, } \\
(\mathrm{MPa})\end{array}$ \\
\hline 1 & 1.91 & 7.76 & 0.00033 \\
2 & 6.83 & 13.06 & 0.00177 \\
3 & 5.01 & 13.56 & 0.00097 \\
4 & 7.30 & 13.08 & 0.00212 \\
5 & 7.88 & 12.51 & 0.00247 \\
6 & 9.87 & 18.06 & 0.00274 \\
7 & 13.62 & 20.60 & 0.00457 \\
8 & 6.48 & 11.13 & 0.00198 \\
9 & 9.45 & 12.00 & 0.00305 \\
\hline
\end{tabular}

where $L$ is the beam span and $I$ is the beam moment of inertia $\left(I=(1 / 12) b h^{3}\right)$. The tensile strain in the lower fiber of the beam under loading was calculated as

$$
\varepsilon=\frac{108}{23} \frac{\delta \cdot h}{L^{2}}
$$

Finally, resilience was measured by calculating the area under the stress versus strain curve.

2.2. Results and Discussion. The average values of the mechanical properties (flexural strength, Young's modulus, and resilience) of the tested beams for each batch are presented in Table 2. The results from the first seven batches show that CNFs are particularly effective in increasing these properties when the cement paste was proportioned to have normal strength $(\mathrm{w} / \mathrm{c}=0.40)$ and when the specimen was exposed to drying condition in the first $24 \mathrm{hrs}$ (a situation that can occur frequently in the concrete construction industry). In this condition, as the comparison of the results from batch 1 and batch 2 shows, the increase in strength due to the utilization of CNFs with the concentration of $1.0 \mathrm{wt} \%$ of cement was more than $250 \%$. In addition, the increases in Young's modulus and resilience were $68 \%$ and $430 \%$, respectively.

When the batches with the same proportions as those of batch 1 and batch 2 were cast and moist-cured in the RH of nearly $100 \%$, the increases in mechanical properties due to the incorporation of CNF are significantly smaller. In fact, the comparison between batches 3 and 4 shows that the increase in strength due to the utilization of CNFs with the concentration of $1.0 \mathrm{wt} \%$ of cement was approximately $45 \%$. There was no increase in Young's modulus, and the increase in resilience was approximately $120 \%$.

The results from the high-strength hardened cement pastes $(\mathrm{w} / \mathrm{c}=0.25)$ and the silica-fume-incorporated cement pastes show similar values of increase in mechanical properties due to the addition of CNFs with the concentration of $1.0 \mathrm{wt} \%$ of cement. In other words, CNFs are most effective when cement paste is proportioned to have normal strength and exposed to drying conditions in the first $24 \mathrm{hrs}$. For highstrength hardened cement paste that was exposed to drying conditions in the first $24 \mathrm{hrs}$ the increase in strength due to the addition of CNFs with the concentration of $1.0 \mathrm{wt} \%$ of 


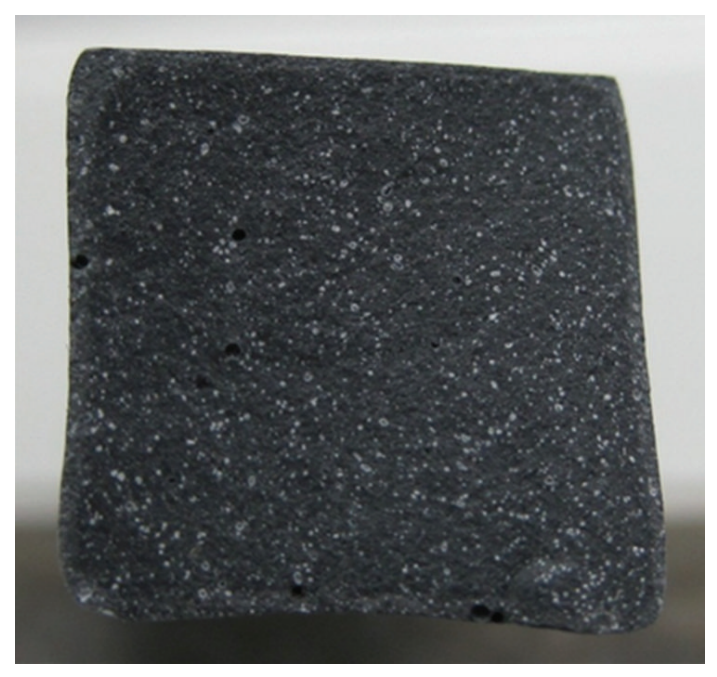

FIGURE 2: Clumps of silica fume particles (lighter colored particles) seen in the fractured surface of a hardened cement paste beam. It is postulated that these clumps generate weak zones that can partially offset the effect of CNFs on mechanical properties. The beam has a cross-section side length of $15.9 \mathrm{~mm}$ (0.625 inch).

cement was 38\%. As for the silica-fume-incorporated cement paste cast and moist cured in RH of $100 \%$, this increase was $45 \%$. This observation is interesting because, although the dispersion of CNFs in the batches with silica fume is more uniform, the increase in flexural strength imparted by CNFs in batches with silica fume is very similar to the increase achieved in the ones without silica fume. A possible reason is the dispersion of silica fume particles within the cementitious matrix. Typically, as-received silica fume contains clumps. These clumps of silica fume particles are large enough to be seen by the naked eye. They form weak zones that can partially counterbalance the effect of CNFs on mechanical properties. More investigations are required to validate this hypothesis (Figure 2).

The results from batch 5 reveal an important fact about the effectiveness of the ultrasonication method for achieving a quality dispersion of CNFs in cement paste. Batch 5 has the same proportions as batch 4 . It is a normal strength cement paste containing CNFs. The only difference is that batch 4 is produced by the ultrasonication method, while in batch 5 CNFs were not initially dispersed in water but were simply added to and mixed with cement paste in the form of dry powder. Interestingly, contrary to what one might presuppose, the mechanical properties of beams made from batches 4 and 5 are similar. In fact, the average flexural strength of the beams made from batch 5 is slightly higher than that of the beams made from batch 4 . The reason, as explained earlier, is that in ultrasonication method, although CNFs are initially dispersed uniformly in water, they reagglomerate when mixed with cement. Therefore, ultrasonication method was not only ineffective, but also the damage to the CNFs caused by ultrasonic processing [9] apparently resulted in a slight reduction in flexural strength.

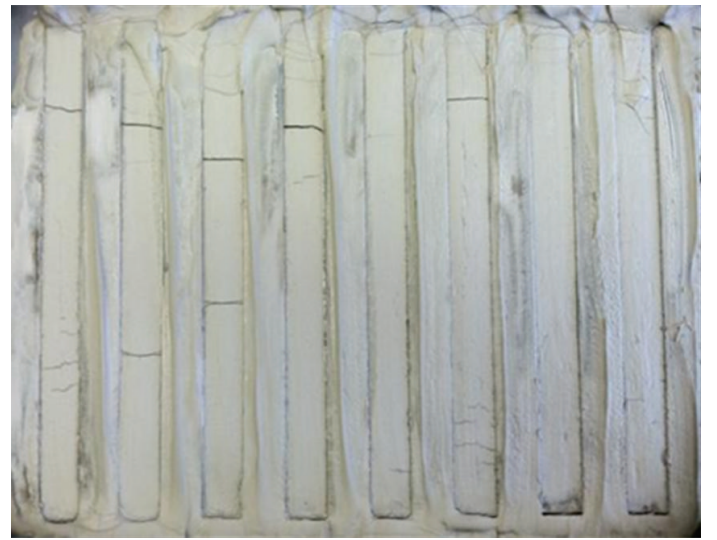

FIGURE 3: Cement paste from batch $6(\mathrm{w} / \mathrm{c}=0.25$, without silica fume and CNF) in the molds $24 \mathrm{hrs}$ after casting. The beams were exposed to drying conditions during this period and formed shrinkage cracks, some of them causing fracture through the depth of the beams before demolding. The beams have a cross-section with the side length of $15.9 \mathrm{~mm}$ ( $0.625 \mathrm{inch})$ and length of $240 \mathrm{~mm}$ (9.5 in).

Observations of shrinkage cracking of the specimens 24 hrs after casting provided a deeper insight into the contribution of CNFs to the mechanical properties and behavior of hardened cement paste. These observations show that when high-strength cement paste $(\mathrm{w} / \mathrm{c}=0.25)$ and the silica-fume-incorporated cement paste are exposed to drying conditions in the first $24 \mathrm{hrs}$, several large cracks form in the beam specimens, particularly in silica-fumeincorporated specimens. These cracks are usually so deep that they fracture the beams into multiple fragments while they are still in the mold.

Figure 3 shows the image of high-strength cement paste $(\mathrm{w} / \mathrm{c}=0.25$, without $\mathrm{CNF})$ beam specimens $24 \mathrm{hrs}$ after casting (batch 6). The top surfaces of these beams were exposed to drying conditions during the first $24 \mathrm{hrs}$. Large cracks on the top surfaces of most of the beams can be seen. Since most of the beams fractured before $24 \mathrm{hrs}$ and could not be tested, an identical batch was made and more beams were produced. The cracking problem did not occur when batch 7 (having same proportions as batch 6, except for incorporating CNFs) was produced in the same conditions. A few extremely shallow cracks were seen on the surface, and when the beams were tested the fracture did not even initiate from any of those cracks. As mentioned earlier, the testing showed that CNFs resulted in an increase of $38 \%$ in the flexural strength of high-strength cement paste. However in light of the mentioned observation, the increase in strength due to the addition of CNFs to the drying cement paste is essentially infinite since most of the beams were broken before demolding, which means that their flexural strength was essentially zero.

Figure 4 shows the image of silica-fume-incorporated beams (without CNF), with the same mix proportions as batch 8 , but exposed to drying condition in the first $24 \mathrm{hrs}$ after casting. Multiple major cracks can be observed in all the beams. The reason for the formation of large cracks 


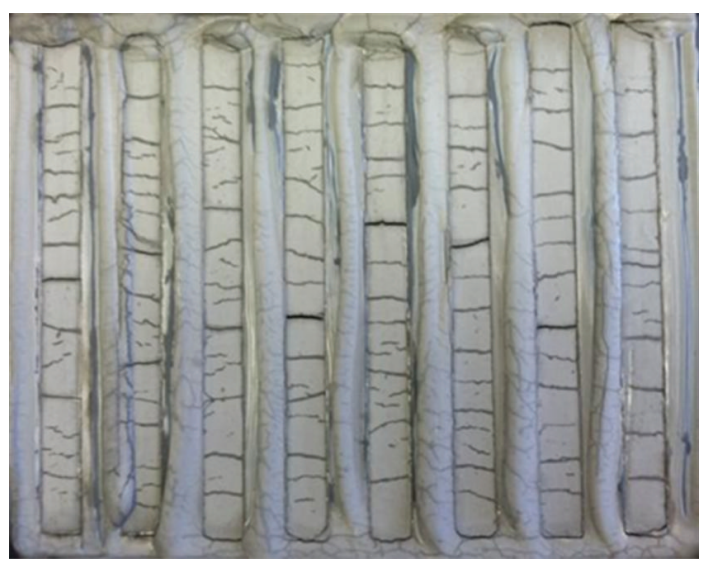

FIgUre 4: Cement paste (w/c $=0.40$, with silica fume to cement ratio of $20 \mathrm{wt} \%$, and without CNF) in the molds $24 \mathrm{hrs}$ after casting. The beams were exposed to drying condition during this period and formed multiple deep shrinkage cracks. All of the beams were fractured into multiple pieces before demolding and therefore none of them could be tested in bending. The beams have a cross-section with the side length of $15.9 \mathrm{~mm}(0.625 \mathrm{inch})$ and length of $240 \mathrm{~mm}$ (9.5 in).

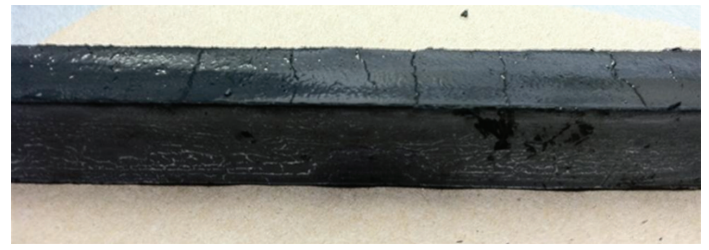

Figure 5: A beam made from batch 9, 24 hrs after casting and immediately after demolding. Batch 9 beams were produced in the same condition (no curing) and with the same mix proportions as those of the beams shown in Figure $4(\mathrm{w} / \mathrm{c}=0.40$, with silica fume to cement ratio of $20 \mathrm{wt} \%$ ). The only difference is that batch 9 contains CNF with the concentration of $1.0 \%$ of cement mass. There are only very shallow shrinkage cracks at the surface. The beams did not break through any of those cracks during bending test.

in low water content and silica-fume-incorporated cement pastes is the presence of a high shrinkage gradient. Both high cement content and silica fume reduce the pore size distribution of cement paste. As a result, the diffusivity of concrete decreases. After casting, the water within the pore network of the top surface of the beams begins to evaporate. However, due to the very low diffusivity, the water from the lower depths of the beam cannot migrate to the top surface and evaporate. Therefore, the top surface of the beam undergoes a remarkable degree of drying shrinkage while such shrinkage does not occur under that surface. That results in a significant shrinkage stress gradient that eventually results in formation of cracks that continue to deepen while the beam keeps shrinking. However, similar to the case of high-strength, low w/c cement paste, the utilization of CNFs essentially eliminates the problem of early age shrinkage cracking (Figure 5).

\section{Concluding Remarks}

In this work, several existing challenges concerning the utilization of carbon nanofilaments in cementitious materials were discussed. Those challenges are related to the poor dispersibility of $\mathrm{CNF} / \mathrm{Ts}$, which can vary depending on the type of the carbon nanofilaments. The ultrasonication method, as the most commonly used procedure for dispersing $\mathrm{CNF} / \mathrm{Ts}$ within cementitious matrices, is not necessarily effective since the $\mathrm{CNF} / \mathrm{Ts}$, which are in part damaged by the ultrasonic processing, tend to reagglomerate when mixed with cement. The utilization of large concentrations of silica fume enhances and stabilizes dispersion of CNF/Ts within cementitious matrices. However, silica fume clumps can generate weak zones that partially offset the effectiveness of $\mathrm{CNF} / \mathrm{Ts}$ for enhancing the mechanical properties of cementitious materials.

An experimental testing program was performed to investigate the effect of CNFs on mechanical behavior and properties of hardened cement paste. The results showed that, in absence of moist curing in the first $24 \mathrm{hrs}$ after mixing the paste, the CNFs increase the flexural strength of hardened cement paste by over $250 \%$. The effect of CNFs on mechanical properties of high-strength paste with either low $\mathrm{w} / \mathrm{c}$ or silica fume was not as large (less than $50 \%$ ).

It was shown that CNFs are effective in preventing shrinkage cracks that occur in cement paste in the absence of moist curing. Both the cement paste with a low w/c and the cement paste containing silica fume have lower porosity and therefore lower diffusivity. That, in absence of moist curing, causes a large shrinkage gradient and therefore stress gradient within the paste placed in molds, which eventually results in the formation of deep cracks. The observations showed that a CNF concentration of $1.0 \%$ by the weight of cement can successfully prevent the formation of such cracks. As a conclusion, it can be stated that, in absence of moist curing, CNFs are very beneficial additives in cement paste even when they are poorly dispersed. They greatly increase the strength of normal-strength hardened cement paste and they mitigate the issue of shrinkage cracking in high-strength hardened cement paste.

\section{References}

[1] J. P. Salvetat and A. J. Kuik, "Electronic and mechanical properties of carbon nanotubes," Advanced Materials, vol. 22, article 7, 1997.

[2] E. W. Wong, P. E. Sheehan, and C. M. Lieber, "Nanobeam mechanics: elasticity, strength, and toughness of nanorods and nanotubes," Science, vol. 277, no. 5334, pp. 1971-1975, 1997.

[3] M. F. Yu, O. Lourie, M. J. Dyer, K. Moloni, T. F. Kelly, and R. S. Ruoff, "Strength and breaking mechanism of multiwalled carbon nanotubes under tensile load," Science, vol. 287, no. 5453, pp. 637-640, 2000.

[4] D. A. Walters, L. M. Ericson, M. J. Casavant et al., "Elastic strain of freely suspended single-wall carbon nanotube ropes," Applied Physics Letters, vol. 74, no. 25, pp. 3803-3805, 1999.

[5] O. Lourie, D. M. Cox, and H. D. Wagner, "Buckling and collapse of embedded carbon nanotubes," Physical Review Letters, vol. 81, no. 8, pp. 1638-1641, 1998. 
[6] B. Yakobson and P. Avouris, "Mechanical properties of carbon nanotubes," in Carbon Nanotubes, Topics in Applied Physics, pp. 287-327, Springer, Berlin, Germany, 2001.

[7] T. Ozkan, Q. Chen, M. Naraghi, and I. Chasiotis, "Mechanical and interface properties of carbon nanofibers for polymer nanocomposites," in Proceedings of the International SAMPE Technical Conference and Exhibition, Multifunctional Materials, Working Smarter Together (SAMPE '08), Nashville, Tenn, USA, September 2008.

[8] A. Yazdanbakhsh, Z. C. Grasley, B. Tyson, and R. K. Abu AlRub, "Carbon nano filaments in cementitious materials: some issues on dispersion and interfacial bond," in Proceedings of the American Concrete Institute (ACI '09), vol. 267, pp. 21-34, November 2009.

[9] A. Yazdanbakhsh, Z. Grasley, B. Tyson, and R. K. Abu AlRub, "Distribution of carbon nanofibers and nanotubes in cementitious composites," Transportation Research Record, no. 2142, pp. 89-95, 2010.

[10] J. Zhao, D. Shi, and J. Lian, "Small angle light scattering study of improved dispersion of carbon nanofibers in water by plasma treatment," Carbon, vol. 47, no. 10, pp. 2329-2336, 2009.

[11] S. Azoubel and S. Magdassi, "The formation of carbon nanotube dispersions by high pressure homogenization and their rapid characterization by analytical centrifuge," Carbon, vol. 48, no. 12, pp. 3346-3352, 2010.

[12] M. Nadler, T. Mahrholz, U. Riedel, C. Schilde, and A. Kwade, "Preparation of colloidal carbon nanotube dispersions and their characterisation using a disc centrifuge," Carbon, vol. 46, no. 11, pp. 1384-1392, 2008.

[13] J. Yu, N. Grossiord, C. E. Koning, and J. Loos, "Controlling the dispersion of multi-wall carbon nanotubes in aqueous surfactant solution," Carbon, vol. 45, no. 3, pp. 618-623, 2007.

[14] Y. Bai, I. S. Park, S. J. Lee et al., "Aqueous dispersion of surfactant-modified multiwalled carbon nanotubes and their application as an antibacterial agent," Carbon, vol. 49, no. 11, pp. 3663-3671, 2011.

[15] H. Leinonen, M. Pettersson, and M. Lajunen, "Water-soluble carbon nanotubes through sugar azide functionalization," Carbon, vol. 49, no. 4, pp. 1299-1304, 2011.

[16] M. J. Sobkowicz, J. R. Dorgan, K. W. Gneshin, A. M. Herring, and J. T. McKinnon, "Controlled dispersion of carbon nanospheres through surface functionalization," Carbon, vol. 47, no. 3, pp. 622-628, 2009.

[17] L. I. Nasibulina, I. V. Anoshkin, S. D. Shandakov et al., "Direct synthesis of carbon nanofibers on cement particles," Transportation Research Record, no. 2142, pp. 96-101, 2010.

[18] J. M. Makar and J. J. Beaudoin, "Carbon nanotubes and their application in the construction industry," in Proceedings of the 1st International Symposium on Nanotechnology in Construction, Paisley, Scotland, 2004.

[19] J. Makar, J. Margeson, and J. Luh, "Carbon nanotube/cement composites - early results and potential applications," in Proceedings of the 3rd International Conference on Construction Materials: Performance, Innovations and Structural Implications, Vancouver, Canada, 2005.

[20] Z. Grasley and A. Yazdanbakhsh, "High-performance stressrelaxing cementitious composites (SRCC) for crack-free pavements and transportation structures," Tech. Rep. DTFH61-08H-00004, Federal Highway Administration, 2011.

[21] P. C. Ma, S. Y. Mo, B. Z. Tang, and J. K. Kim, "Dispersion, interfacial interaction and re-agglomeration of functionalized carbon nanotubes in epoxy composites," Carbon, vol. 48, no. 6, pp. 1824-1834, 2010.
[22] M. Kotaki, K. Wang, M. L. Toh, L. Chen, S. Y. Wong, and C. He, "Electrically conductive epoxy/clay/vapor grown carbon fiber hybrids," Macromolecules, vol. 39, no. 3, pp. 908-911, 2006.

[23] J. F. Feller, S. Bruzaud, and Y. Grohens, "Influence of clay nanofiller on electrical and rheological properties of conductive polymer composite," Materials Letters, vol. 58, no. 5, pp. 739-745, 2004.

[24] L. Liu and J. C. Grunlan, "Clay assisted dispersion of carbon nanotubes in conductive epoxy nanocomposites," Advanced Functional Materials, vol. 17, no. 14, pp. 2343-2348, 2007.

[25] F. Sanchez and C. Ince, "Microstructure and macroscopic properties of hybrid carbon nanofiber/silica fume cement composites," Composites Science and Technology, vol. 69, no. 7-8, pp. 1310-1318, 2009.

[26] X. Fu and D. D. L. Chung, "Submicron-diameter-carbonfilament cement-matrix composites," Carbon, vol. 36, no. 4, pp. 459-462, 1998.

[27] S. Luo, T. Liu, and B. Wang, "Comparison of ultrasonication and microfluidization for high throughput and large-scale processing of SWCNT dispersions," Carbon, vol. 48, no. 10, pp. 2992-2994, 2010.

[28] R. Ormsby, T. McNally, C. Mitchell et al., "Effect of MWCNT addition on the thermal and rheological properties of polymethyl methacrylate bone cement," Carbon, vol. 49, no. 9, pp. 2893-2904, 2011.

[29] P. Fidjestol, "Guide for the use of silica fume in concrete," ACI Committee 234 Reports, 2006.

[30] T. Kowald, "Influence of surface-modified carbon nanotubes on ultrahigh performance concrete," in Proceedings of the International Symposium on Ultra High Performance Concrete, 2004.

[31] G. Y. Li, P. M. Wang, and X. Zhao, "Mechanical behavior and microstructure of cement composites incorporating surfacetreated multi-walled carbon nanotubes," Carbon, vol. 43, no. 6, pp. 1239-1245, 2005.

[32] C. Gay and F. Sanchez, "Performance of carbon nanofibercement composites with a high-range water reducer," Transportation Research Record, no. 2142, pp. 109-113, 2010.

[33] Z. S. Metaxa, M. S. Konsta-Gdoutos, and S. P. Shah, "Carbon nanotubes reinforced concrete," in Proceedings of the American Concrete Institute (ACI '09), pp. 11-20, New Orleans, La, USA, November 2009.

[34] S. P. Shah, "Nanoscale modification of cementitious materials," in Proceedings of the 3rd International Symposium on Nanotechnology in Construction, pp. 125-130, 2009.

[35] M. S. Konsta-Gdoutos, Z. S. Metaxa, and S. P. Shah, "Highly dispersed carbon nanotube reinforced cement based materials," Cement and Concrete Research, vol. 40, no. 7, pp. 10521059, 2010.

[36] M. S. Konsta-Gdoutos, Z. S. Metaxa, and S. P. Shah, "Multiscale mechanical and fracture characteristics and early-age strain capacity of high performance carbon nanotube/cement nanocomposites," Cement and Concrete Composites, vol. 32, no. 2, pp. 110-115, 2010.

[37] A. Cwirzen, K. Habermehl-Cwirzen, and V. Penttala, "Surface decoration of carbon nanotubes and mechanical properties of cement/carbon nanotube composites," Advances in Cement Research, vol. 20, no. 2, pp. 65-73, 2008.

[38] B. M. Tyson, R. K. Abu Al-Rub, A. Yazdanbakhsh, and Z. Grasley, "Carbon nanotubes and carbon nanofibers for enhancing the mechanical properties of nanocomposite cementitious materials," Journal of Materials in Civil Engineering, vol. 23, no. 7, pp. 1028-1035, 2011. 

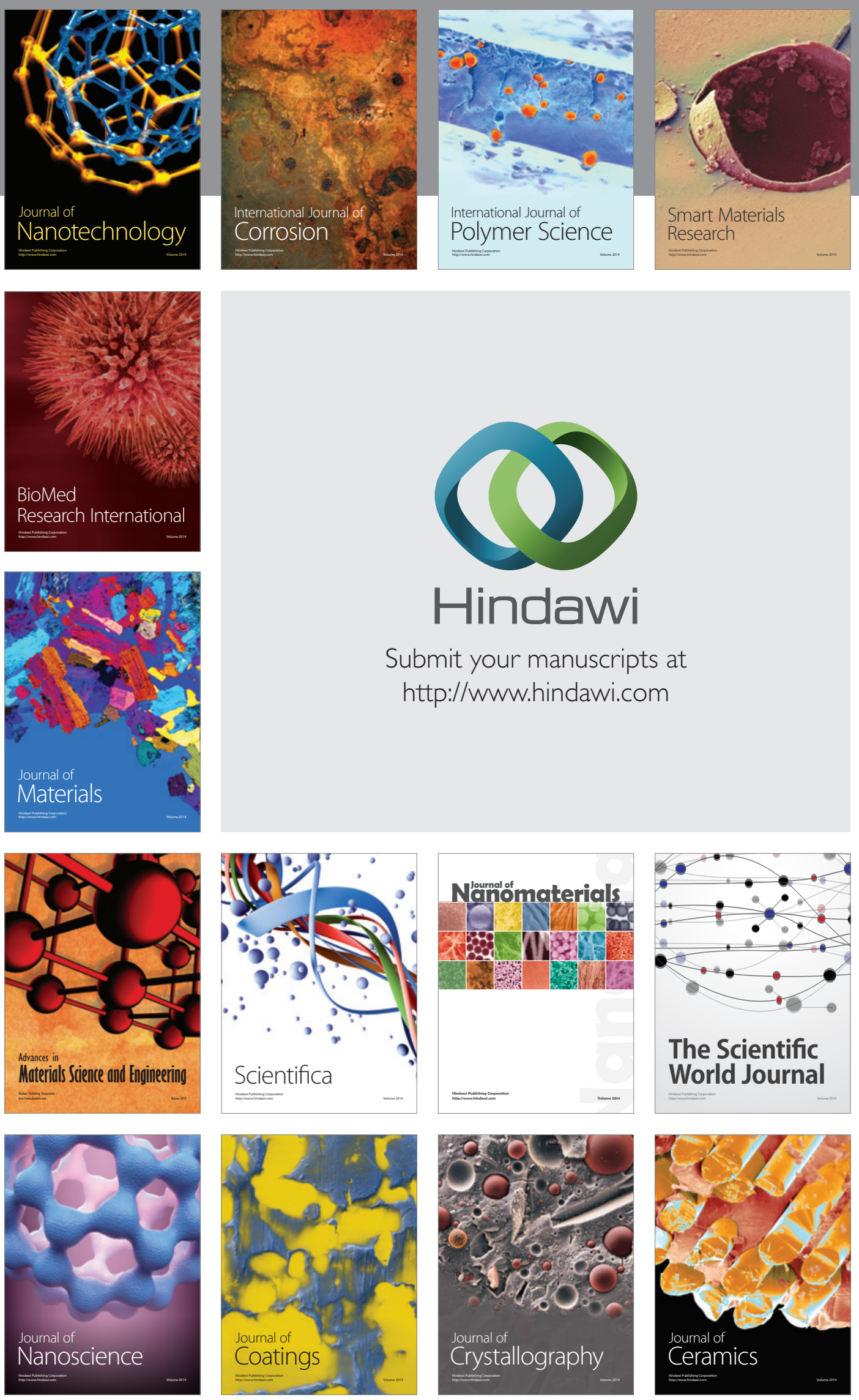

The Scientific World Journal

Submit your manuscripts at

http://www.hindawi.com

\section{World Journal}

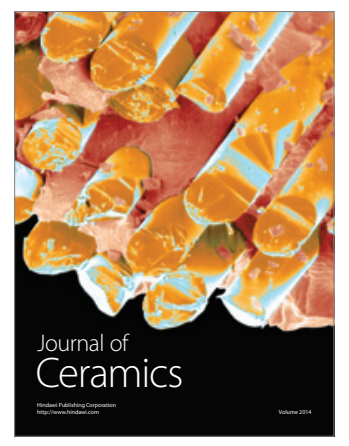

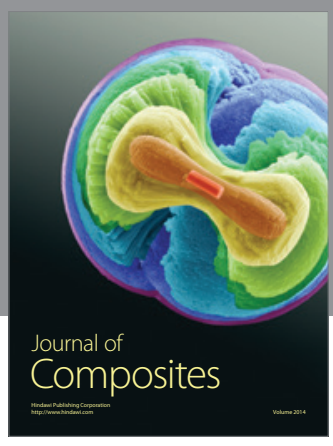
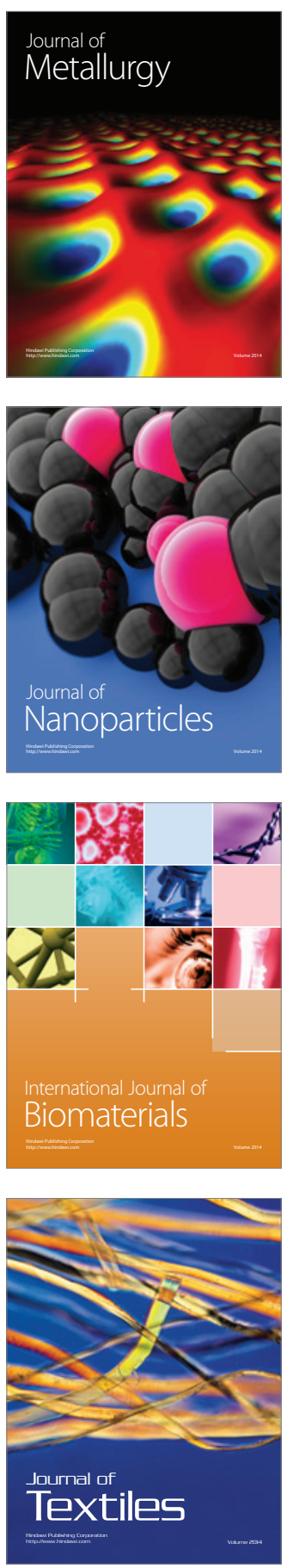